Physical Review A 69, 032318 (2004)

\title{
Optical Generation and Quantitative Characterizations of Electron-hole Entanglement
}

\author{
$\mathrm{Yu}$ Shi \\ Cavendish Laboratory, University of Cambridge, Cambridge CB3 OHE, United Kingdom and \\ Department of Physics, University of Illinois at Urbana-Champaign, Urbana, IL 61801, USA
}

\begin{abstract}
Using a method of characterizing entanglement in the framework of quantum field theory, we investigate the optical generation and quantitative characterizations of quantum entanglement in an electron-hole system, in presence of spin-orbit coupling, and especially make a theoretical analysis of a recent experimental result. Basically, such entanglement should be considered as between occupation numbers of single particle basis states, and is essentially generated by coupling between different single particle basis states in the second quantized Hamiltonian. Interaction with two resonant light modes of different circular polarizations generically leads to a superposition of ground state and two heavy-hole excitonic states. When and only when the state is a superposition of only the two excitonic eigenstates, the entanglement reduces to that between two distinguishable particles, each with two degrees of freedom, namely, band index, as characterized by angular momentum, and orbit, as characterized by position or momentum. The band-index state, obtained by tracing over the orbital degree of freedom, is found to be a pure state, hence the band-index and orbital degrees of freedom are separated in this state. We propose some basic ideas on spatially separating the electron and the hole, so that the entanglement of band-indices, or angular momenta, is between spatially separated electron and hole.
\end{abstract}

PACS numbers: 03.67.Mn, 71.35.-y

\section{INTRODUCTION}

As an essential quantum characteristic, quantum entanglement refers to non-factorization of the state of a composite system in terms of states of subsystems [1]. It is of fundamental importance for quantum information and quantum foundations [2]. In addition to such systems as photons, atoms and trapped ions, large amount of work is also going on in generating entanglement in condensed matter systems. Investigations are made on generating and separating entangled electron-electron [3] or electron-hole [4, 5, 6, 7] pairs in solid states. There is also a lot of proposals of using excitons for quantum information processing $8,[9,[10]$. Very recently, coherent optical control of a biexciton in a quantum dot is also reported [1]. It should be noted however that microscopic electronic entanglement is ubiquitous in many-electron systems and is closely related to the physical properties of condensed matter [12]. Nevertheless, current researches on entanglement generation in solid states, largely in mesoscopic systems, have some special merits or aims such as controllability and spatial separation.

For an existing electron-hole pair, one can directly study their entanglement by using the well-known method for distinguishable particles. However, this approach has limited validity. This is because electron-hole pairs are excitations, or quasi-particles, of the manyparticle system, and one needs to consider larger Hilbert space when their creation and annihilation are involved. For example, the state generated in [4], as well as the state in the proposal in [6], have a ground state component, in which there is no excited electron or hole at all. Hence an explanation based on the approach of entanglement of distinguishable particles is not sufficient.
Therefore we need to understand entanglement in the framework of quantum field theory. Such an approach was made in [12], where it was applied to investigate entanglement in many-particle physics. For a system of identical particles, entanglement, as the correlation beyond permutation symmetry, can be defined in terms of occupation numbers of different single particle basis states or modes 13, 14, 15. To generate occupationnumber entanglement, it is the coupling between different single particle basis states, rather than interaction between particles, that is essential. We would like to note that photon entanglement 16] is fundamentally also occupation-number entanglement, and is thus generated by the mode-mode coupling. The usual description in terms of distinguishable particles is valid only as a limiting case, because there is a degree of freedom, e.g. the direction of movement, effectively distinguishes the photons, and they become distinguished after separation.

Electron-hole entanglement is basically an occupationnumber entanglement in the many-electron system, and can be simplified to entanglement between distinguishable quasi-particles when and only when there is one electron and one hole in each component of the state. Indeed, it can be seen that mode-mode coupling underlies the entanglement generation in [4, [5, 6 ]. In the situation studied by [7], the mode corresponds to the position, hence entanglement can be caused merely by the hopping.

In this paper, we make a theoretical account of the physics underlying the excellent experimental result in 4], and make a detailed analysis on the entanglement and its generation in this electron-hole system, with the spinorbit coupling taken into account. Basically, the state generated by the coupling with the two laser fields of different circular polarizations is a coherent superposition of 
the ground state and two excitonic eigenstates. Coulomb interaction makes the biexcitonic state off-resonant, but this is irrelevant to the necessity of interaction of particles in generating entanglement between distinguishable particles. Occupation-number entanglement is still generated if the Coulomb interaction is negligible, and if only one light mode is present. When the state does not have the ground state component, the superposition of the two excitonic eigenstates can be described in terms of two distinguishable particles. Interestingly, the bandindex state, as obtained by tracing out the orbital degrees of freedom, i.e. the Bloch wavevectors or the positions, is found to be a pure state in this case. We briefly propose several methods to spatially separate the electron and the hole, making band-index entanglement (i.e. entanglement in angular momenta and in effective masses) nonlocal in positions.

The rest of this article is organized in the following way. In Sec. II, we make an introduction to the method of entanglement characterization in the framework of quantum field theory. In Sec. III, as a preliminary, we discuss electron-hole entanglement in absence of spin-orbit coupling. Then in Sec. IV, using field theory, we give the theoretical account of the physical process underlying the experiment in Ref. [4]. The entanglement in the resulting state is characterized in Sec. V. Some basic ideas about spatially separating the electron and hole are described in Sec. VI. A summary is made in Sec. VII.

\section{ENTANGLEMENT IN QUANTUM FIELD THEORY}

We first make an overview of the method of entanglement characterization in quantum field theory, in the setting of condensed matter physics 12 .

In terms of occupation numbers of single particle states for a chosen single particle basis, a many-particle state can be expressed as

$$
|\psi\rangle=\sum_{n_{1}, \cdots, n_{\infty}} f\left(n_{1}, \cdots, n_{\infty}\right)\left|n_{1}, \cdots, n_{\infty}\right\rangle
$$

where $n_{i}$ is the occupation number of single particle state $i,\left|n_{1}, \cdots, n_{\infty}\right\rangle \equiv a_{1}^{\dagger n_{1}} \cdots a_{\infty}^{\dagger}{ }^{n_{\infty}}|0\rangle$.

Choosing a different single particle basis means partitioning the system into a different set of subsystems, based on which the entanglement is then defined. But once a single particle basis is chosen, the entanglement is invariant under any unitary operation on individual single particle basis states, i.e. when there is no coupling between different single particle basis states. In other words, in the present case, the meaning of "local operations" as previously used in quantum information theory is generalized to operations on the corresponding single particle basis states, as indexed by the subscript $i$ above. Of course, it is constrained that some kinds of generalized "local" unitary operations do not exist physically.
Once this generalization of the meaning of subsystems and local operations is made, the usual method of calculating the amount of entanglement, as developed in quantum information theory can be applied. Quantitatively, one considers the Fock-state reduced density matrix of a set of single particle basis states $1, \cdots, l$,

$$
\begin{gathered}
\left\langle n_{1}^{\prime}, \cdots, n_{l}^{\prime}\left|\rho_{l}(1 \cdots l)\right| n_{1}, \cdots, n_{l}\right\rangle \equiv \\
\sum_{n_{l+1}, \cdots, n_{\infty}}\left\langle n_{1}^{\prime}, \cdots, n_{l}^{\prime}, n_{l+1}, n_{\infty}|\rho| n_{1}, \cdots, n_{l}, n_{l+1}, n_{\infty}\right\rangle .
\end{gathered}
$$

Its von Neumann entropy measures the entanglement of this set of single particle basis states and the rest of the system. This is a use of the well-known result for a pure state of a composite system, the entanglement between a subsystem $A$ and the rest of the system is quantified as the von Neumann entropy of the reduced density matrix of $A, S_{A}=-t r_{A} \rho_{A} \ln \rho_{A}$ [17].

One can also define the entanglement relative to the ground state $|G\rangle$, by only considering the excited particles. Then $n_{i}$ in (2) is understood as the number of the excited particles, which are absent in the ground state $|G\rangle$, i.e. $\left|n_{1}, \cdots, n_{\infty}\right\rangle \equiv a_{1}^{\dagger_{1}} \cdots a_{\infty}^{\dagger}{ }^{n_{\infty}}|G\rangle$.

Now we proceed to dynamics. In general, for a system with two subsystems $A$ and $B$, the Hamiltonian is always of the form

$$
H=H_{A}+H_{B}+H_{A B},
$$

where $H_{A}$ only acts on $A, H_{B}$ only acts on $B$, while $H_{A B}$ acts on both $A$ and $B$. If $H_{A B}=0$, then an initial non-entangled state $\left|\phi_{A}\right\rangle \otimes\left|\phi_{B}\right\rangle$ evolves to $\exp \left(-i H_{A} t\right)\left|\phi_{A}\right\rangle \otimes \exp \left(-i H_{B} t\right)\left|\phi_{B}\right\rangle$ at any time $t$, which is still non-entangled. Hence the coupling term $H_{A B}$ is necessary for entanglement generation. For two distinguishable particles, $A$ and $B$ can directly represent these two particles.

Here we consider the non-relativistic field theory. The Hamiltonian is

$$
\begin{aligned}
\mathcal{H} & =\int d^{3} r \hat{\psi}^{\dagger}(\mathbf{r}) h(\mathbf{r}) \hat{\psi}(\mathbf{r})+\int d^{3} r \hat{\psi}^{\dagger}(\mathbf{r}) h^{\prime}(\mathbf{r}) \hat{\psi}(\mathbf{r}) \\
& +\frac{1}{2} \int d^{3} r \int d^{3} r^{\prime} \hat{\psi}^{\dagger}(\mathbf{r}) \hat{\psi}^{\dagger}\left(\mathbf{r}^{\prime}\right) V\left(\mathbf{r}, \mathbf{r}^{\prime}\right) \hat{\psi}\left(\mathbf{r}^{\prime}\right) \hat{\psi}(\mathbf{r}),
\end{aligned}
$$

where $h(\mathbf{r})$ is the single particle Hamiltonian including the kinetic energy, $h^{\prime}(\mathbf{r})$ is some external potential which is not included in $h(\mathbf{r})$, for example, the coupling with electromagnetic field, and $V\left(\mathbf{r}, \mathbf{r}^{\prime}\right)$ is the particle-particle interaction. The reason for separating $h^{\prime}$ from $h$ will be clear below. The field operator $\hat{\psi}(\mathbf{r})$ can be expanded in an arbitrarily chosen single particle basis as $\hat{\psi}(\mathbf{r})=$ $\sum_{i} \phi_{i}(\mathbf{r}) a_{i}$, where $i$ is the collective index of the single particle state, which may include spin if needed, $\phi_{i}(\mathbf{r})$ is the single particle wavefunction in position space.

In the form of (3), the Hamiltonian can be written as

$$
\begin{aligned}
\mathcal{H}= & \sum_{i j}\langle i|h| j\rangle a_{i}^{\dagger} a_{j} \\
& +\sum_{i j}\left\langle i\left|h^{\prime}\right| j\right\rangle a_{i}^{\dagger} a_{j}+\frac{1}{2} \sum_{i j l m}\langle i j|V| l m\rangle a_{i}^{\dagger} a_{j}^{\dagger} a_{m} a_{l},
\end{aligned}
$$


Thus the index $i$, denoting single particle basis states, defines distinguishable subsystems.

Now that the entanglement is that between single particle basis states, its generation needs, in the Hamiltonian $\mathcal{H}$, coupling between different single particle basis states. Therefore, even if $V=0$ and $h^{\prime}=0$, as far as $\langle i|h| j\rangle \neq 0$, $\mathcal{H}$ can still generate occupation-number entanglement between single particle basis state $i$ and $j$. Examples of this case include the tunnelling problem and hopping between Wannier basis states.

However, in many cases, the single particle state is defined by the eigenstates of $h$. For example, electrons and holes corresponding to band structure, i.e. Bloch states. In this single particle basis, which we call proper single particle basis, $h \phi_{\mu}=\epsilon_{\mu} \phi_{\mu}, \int d^{3} r \hat{\psi}^{\dagger}(\mathbf{r}) h(\mathbf{r}) \hat{\psi}(\mathbf{r})=$ $\sum_{\mu} \epsilon_{\mu} a_{\mu}^{\dagger} a_{\mu}$, whose eigenstates are of the form $\otimes_{\mu}\left|n_{\mu}\right\rangle$, where $\mu$ is the collective index of the proper single particle basis. Therefore in the proper single particle basis, entanglement can only be caused by $h^{\prime}$ or by $V$ if they couple different modes. Note that only $V$ is particleparticle interaction.

When there are more than one index in the single particle basis, one of the indices can be used as the tag effectively distinguishing the particles, and the other indices determine whether they are entangled in these degrees of freedom. With this effective distinguishability, the state in the configuration space of the remaining degrees of freedom can be directly obtained from the second-quantized state. For example, in $\frac{1}{\sqrt{2}}\left(a_{\mathbf{k}^{\prime} \uparrow}^{\dagger} a_{\mathbf{k} \downarrow}^{\dagger}+\right.$ $\left.a_{\mathbf{k}^{\prime} \downarrow}^{\dagger} a_{\mathbf{k} \uparrow}^{\dagger}\right)|0\rangle$, where $\mathbf{k}^{\prime}$ and $\mathbf{k}$ represent momenta, one can say that the particle in $\left|\mathbf{k}^{\prime}\right\rangle$ and the particle in $|\mathbf{k}\rangle$ are spin-entangled. One can also say that the particle in $|\uparrow\rangle$ and the particle in $|\downarrow\rangle$ are momentum-entangled. With the momentum as the distinguishing tag, the state can be written as $\frac{1}{\sqrt{2}}\left(|\uparrow\rangle_{\mathbf{k}^{\prime}}|\downarrow\rangle_{\mathbf{k}}+|\uparrow\rangle_{\mathbf{k}^{\prime}}|\downarrow\rangle_{\mathbf{k}}\right)$, with spin entanglement. Alternatively, with the spin as the distinguishing tag, the state can be written as $\frac{1}{\sqrt{2}}\left(\left|\mathbf{k}^{\prime}\right\rangle_{\uparrow}|\mathbf{k}\rangle_{\downarrow}+|\mathbf{k}\rangle_{\uparrow}\left|\mathbf{k}^{\prime}\right\rangle_{\downarrow}\right)$, with momentum entanglement.

\section{ELECTRON-HOLE ENTANGLEMENT IN ABSENCE OF SPIN-ORBIT COUPLING}

The ground state of an electron gas is $|G\rangle=$ $\prod_{\mathbf{k}}^{|\mathbf{k}|<k_{F}} a_{\mathbf{k} \uparrow}^{\dagger} a_{\mathbf{k} \downarrow}^{\dagger}|0\rangle$, where $k_{F}$ is the Fermi momentum. This is clearly a non-entangled state. One can introduce the hole operator $b_{\mathbf{k} s}^{\dagger}=a_{-\mathbf{k}-s}$ for $|\mathbf{k}|<k_{F}$ (we use $s$ and $-s$ to represent the two spin states). An excited state is obtained by creating particle-hole pairs from the ground state. The state $a_{\mathbf{k} s}^{\dagger} b_{\mathbf{k}^{\prime} s^{\prime}}^{\dagger}|G\rangle$, with $|\mathbf{k}|>k_{F}>\left|\mathbf{k}^{\prime}\right|$, is separable. But there is maximal entanglement in state $\frac{1}{\sqrt{2}}\left(a_{\mathbf{k}_{\uparrow}}^{\dagger} b_{\mathbf{k}^{\prime} \downarrow}^{\dagger}+a_{\mathbf{k}_{\downarrow} \downarrow}^{\dagger} b_{\mathbf{k}^{\prime} \uparrow}^{\dagger}\right)|G\rangle$. This state can be written as $\frac{1}{\sqrt{2}}\left(a_{\mathbf{k} \uparrow}^{\dagger} a_{-\mathbf{k}^{\prime} \downarrow}^{\dagger}+a_{\mathbf{k} \downarrow}^{\dagger} a_{-\mathbf{k}^{\prime} \uparrow}^{\dagger}\right) \prod_{|\mathbf{p}|<k_{F}}^{\mathbf{p} \neq-\mathbf{k}^{\prime}} a_{\mathbf{p} \uparrow}^{\dagger} a_{\mathbf{p} \downarrow}^{\dagger} \downarrow|0\rangle$, from which it can be seen that with respect to the empty state, the entanglement is between the excited electron state and the one in the same level as the emptied state but with opposite spin. But with respect to the ground state, it is simply electron-hole entanglement. An electron and a hole, by definition, correspond to different single particle states, and can be regarded as distinguishable particles.

Consider one electron is excited from a valence band to a conduction band. An eigenstate of this excitation, an exciton, in the absence of spin-orbit coupling, is

$$
\sum_{\mathbf{k}, \mathbf{k}^{\prime}} A_{\mathbf{k}, \mathbf{k}^{\prime}}\left|S, S_{z}\right\rangle_{\mathbf{k}, \mathbf{k}^{\prime}}
$$

where $\left|S, S_{z}\right\rangle_{\mathbf{k}, \mathbf{k}^{\prime}}$ represents three triplet states as the ground states, $|1,1\rangle_{\mathbf{k}, \mathbf{k}^{\prime}}=a_{\mathbf{k} \uparrow}^{\dagger} b_{\mathbf{k}^{\prime} \uparrow}^{\dagger}|G\rangle$, $|1,0\rangle_{\mathbf{k}^{\prime}}=\frac{1}{\sqrt{2}}\left(a_{\mathbf{k} \uparrow}^{\dagger} b_{\mathbf{k}^{\prime} \downarrow}^{\dagger}-a_{\mathbf{k} \downarrow}^{\dagger} b_{\mathbf{k}^{\prime} \uparrow}^{\dagger}\right)|G\rangle \quad$ and $|1,-1\rangle_{\mathbf{k}, \mathbf{k}^{\prime}}=a_{\mathbf{k}_{\downarrow}}^{\dagger} b_{\mathbf{k}^{\prime} \downarrow}^{\dagger}|G\rangle$, and one singlet state $|0,0\rangle_{\mathbf{k}, \mathbf{k}^{\prime}}=\frac{1}{\sqrt{2}}\left(a_{\mathbf{k} \uparrow}^{\dagger} b_{\mathbf{k}^{\prime} \downarrow}^{\dagger}+a_{\mathbf{k} \downarrow}^{\dagger} b_{\mathbf{k}^{\prime} \uparrow}^{\dagger}\right)|G\rangle . \quad A_{\mathbf{k}, \mathbf{k}^{\prime}}$ is determined by the Schrödinger equation in momentum representation, $\left(E_{0 c}+\hbar^{2} \mathbf{k}^{2} / 2 m_{e}+E_{0 v}+\hbar^{2} \mathbf{k}^{2} / 2 m_{h}-\right.$ E) $A_{\mathbf{k}, \mathbf{k}^{\prime}}-\sum_{\mathbf{q}, \mathbf{q}^{\prime}}\left(V_{\mathbf{k}-\mathbf{k}^{\prime}-\mathbf{q}^{\prime} \mathbf{q}}^{c v v}-V_{\mathbf{k}-\mathbf{q}^{\prime} \mathbf{q}-\mathbf{k}^{\prime}}^{c c v}\right) A_{\mathbf{q}, \mathbf{q}^{\prime}}=0$, where $E_{0 c}$ is the bottom of the conduction electron band, $E_{0 v}$ is the top of the valence hole band, $V_{\mathbf{k}_{1} \mathbf{k}_{2} \mathbf{k}_{3} \mathbf{k}_{4}}^{\mu \nu \sigma \delta}=$ $\int \phi_{\mu \mathbf{k}_{1}}(\mathbf{r}) \phi_{\nu \mathbf{k}_{2}}\left(\mathbf{r}^{\prime}\right) V\left(\mathbf{r}-\mathbf{r}^{\prime}\right) \phi_{\sigma \mathbf{k}_{3}}\left(\mathbf{r}^{\prime}\right) \phi_{\delta \mathbf{k}_{4}}(\mathbf{r}) d^{3} \mathbf{r} d^{3} \mathbf{r}^{\prime}, \mu, \nu$, $\sigma$ and $\delta$ represent band indices.

Consider $\sum_{\mathbf{k}, \mathbf{k}^{\prime}} A_{\mathbf{k}, \mathbf{k}^{\prime}} \frac{1}{\sqrt{2}}\left(a_{\mathbf{k}_{\uparrow} \uparrow}^{\dagger} b_{\mathbf{k}^{\prime} \downarrow}^{\dagger} \pm a_{\mathbf{k}_{\downarrow}}^{\dagger} b_{\mathbf{k}^{\prime} \uparrow}^{\dagger}\right)|G\rangle$. The occupation-number entanglement between the electron basis state $|\mathbf{k}, \uparrow(\downarrow)\rangle_{e}$ and the rest of the system is $-\left(\alpha_{\mathbf{k}} / 2\right) \ln \left(\alpha_{\mathbf{k}} / 2\right)-\left(1-\alpha_{\mathbf{k}} / 2\right) \ln \left(1-\alpha_{\mathbf{k}} / 2\right)$, where $\alpha_{\mathbf{k}^{\prime}}=$ $\sum_{\mathbf{k}}\left|A_{\mathbf{k}, \mathbf{k}^{\prime}}\right|^{2}$. The occupation-number entanglement between the hole basis state $\left|\mathbf{k}^{\prime}, \downarrow(\uparrow)\right\rangle_{h}$ and the rest of the system is $-\left(\alpha_{\mathbf{k}^{\prime}} / 2\right) \ln \left(\alpha_{\mathbf{k}^{\prime}} / 2\right)-\left(1-\alpha_{\mathbf{k}^{\prime}} / 2\right) \ln \left(1-\alpha_{\mathbf{k}^{\prime}} / 2\right)$.

On the other hand, because the electron and the hole are effectively distinguishable, these states can be written, in the configuration space, as

$$
\sum_{\mathbf{k}, \mathbf{k}^{\prime}} A_{\mathbf{k}, \mathbf{k}^{\prime}}|\mathbf{k}\rangle\left|\mathbf{k}^{\prime}\right\rangle\left|S, S_{z}\right\rangle
$$

So the orbital and spin degrees of freedom are separated, as consistent with the presumption that spin-orbit coupling is neglected. The entanglement in the spin state $\left|S, S_{z}\right\rangle$ is well-known.

But note that when a state is a superposition of ground state, where occupation-numbers of the relevant electron and hole states are zero, and excitonic states, the entanglement cannot be treated as entanglement between distinguishable particles.

An electron-hole pair can be generated by electronlight coupling, which corresponds to $h^{\prime}$ in (5). This underlies the experimental result in [4]. A theoretical study is made below, with spin-orbit coupling taken into account.

\section{PHYSICAL PROCESS WITH SPIN-ORBIT COUPLING}

Now we study the physical process underlying the experiment in [4]. We shall consider coupling with lights 
which are only relevant to the two conduction bands $c 1$ and $c 2$, and the two heavy-hole bands $h 1$ and $h 2$. For these four bands, the total angular momentum $\left(j, m_{j}\right)$ is, respectively, $\left(\frac{1}{2}, \frac{1}{2}\right),\left(\frac{1}{2},-\frac{1}{2}\right),\left(\frac{3}{2}, \frac{3}{2}\right),\left(\frac{3}{2},-\frac{3}{2}\right)$. The neglect of other bands only affects the microscopic expressions of some effective parameters, and the ground state energy, which is not relevant. This band structure is a consequence of spin-orbit coupling, i.e. the spin-orbit coupling has been included in the one-particle Hamiltonian $h(\mathbf{r})$ as in Eq. (4). The field operator $\hat{\psi}(\mathbf{r})$ can be expanded as $\sum_{i \mathbf{k}}\left[a_{i \mathbf{k}} \phi_{c i \mathbf{k}}(\mathbf{r})+b_{i-\mathbf{k}}^{\dagger} \phi_{h i \mathbf{k}}(\mathbf{r})\right]$, where $i=1,2$, $a_{i \mathbf{k}}$ is the electron annihilation operator for the band $c i$, $b_{i-\mathbf{k}} \equiv a_{i \mathbf{k}}^{\dagger}$ is the hole annihilation operator for band $h i$. Consequently, the field theoretical Hamiltonian $\mathcal{H}_{e}$ is reduced to

$$
\begin{gathered}
\mathcal{H}_{e}=E_{0}+\sum_{i} \sum_{\mathbf{k}} E_{c i \mathbf{k}} a_{i \mathbf{k}}^{\dagger} a_{i \mathbf{k}}+\sum_{i} \sum_{\mathbf{k}} E_{h i \mathbf{k}} b_{i \mathbf{k}}^{\dagger} b_{i \mathbf{k}} \\
+\frac{1}{2} \sum_{i j} \sum_{\mathbf{k}_{\mu} \mathbf{k}_{\nu} \mathbf{k}_{\sigma} \mathbf{k}_{\delta}} V_{\mathbf{k}_{\mu} \mathbf{k}_{\nu} \mathbf{k}_{\sigma} \mathbf{k}_{\delta}}^{c i c j c i} a_{i \mathbf{k}_{\mu}}^{\dagger} a_{j \mathbf{k}_{\nu}}^{\dagger} a_{j \mathbf{k}_{\sigma}} a_{i \mathbf{k}_{\delta}} \\
+\frac{1}{2} \sum_{i j} \sum_{\mathbf{k}_{\mu} \mathbf{k}_{\nu} \mathbf{k}_{\sigma} \mathbf{k}_{\delta}} V_{-\mathbf{k}_{\mu}-\mathbf{k}_{\nu}-\mathbf{k}_{\sigma}-\mathbf{k}_{\delta}}^{h i h j h \mathbf{k}_{\delta}} b_{j \mathbf{k}_{\sigma}}^{\dagger} b_{j \mathbf{k}_{\nu}} b_{i \mathbf{k}_{\mu}}- \\
\sum_{i j \mathbf{k}_{\mu} \mathbf{k}_{\nu} \mathbf{k}_{\sigma} \mathbf{k}_{\delta}} \sum_{\mathbf{k}_{\mu}-\mathbf{k}_{\nu}-\mathbf{k}_{\sigma} \mathbf{k}_{\delta}}{ }^{c i h j h j i} V_{\mathbf{k}_{\mu}-\mathbf{k}_{\nu} \mathbf{k}_{\delta}-\mathbf{k}_{\sigma}}^{c i h j c i h j} a_{i \mathbf{k}_{\mu}}^{\dagger} a_{i \mathbf{k}_{\delta}} b_{j \mathbf{k}_{\sigma}}^{\dagger} b_{j \mathbf{k}_{\nu}},
\end{gathered}
$$

where $i=1,2, j=1,2, E_{0}$ is the ground state energy, $V_{\mathbf{k}_{\mu} \mathbf{k}_{\nu} \mathbf{k}_{\sigma} \mathbf{k}_{\delta}}^{\mu \nu \sigma \delta}=\int d^{3} r d^{3} r^{\prime} \phi_{\mu \mathbf{k}_{\mu}}^{*}(\mathbf{r}) \phi_{\nu \mathbf{k}_{\nu}}^{*}\left(\mathbf{r}^{\prime}\right) V(\mathbf{r}-$ $\left.\mathbf{r}^{\prime}\right) \phi_{\sigma \mathbf{k}_{\sigma}}\left(\mathbf{r}^{\prime}\right) \phi_{\delta \mathbf{k}_{\delta}}(\mathbf{r})$ is the matrix element of Coulomb interaction $V$ between single particle states indexed by band index and Bloch wavevector. Originally there is degeneracy between the two conduction bands and between the two heavy-hole bands. But in accordance with the experiment [4], here it is assumed that the degeneracy has been removed by coupling with a perturbative magnetic field, which is included in the single particle Hamiltonian, which also includes the kinetic energy and spin-orbit coupling. The lifting of degeneracy allows the use of effective mass theory for non-degenerate bands, which says $E_{c i \mathbf{k}}=E_{c i 0}+\hbar^{2} \mathbf{k}^{2} / 2 m_{c i}$ and $E_{h i \mathbf{k}}=E_{h i 0}+\hbar^{2} \mathbf{k}^{2} / 2 m_{h i}$, where $m_{c i}$ and $m_{h i}$ are effective masses.

Taking into consideration the angular momentum selection rule in their generation, the relevant low-lying eigenstates of $\mathcal{H}_{e}$ are the following: First, the ground state $|G\rangle$; second, the single-exciton state made up of an electron in $c 1$ band and a hole in $h 1$ band,

$$
\begin{aligned}
|S 1\rangle & =\sum_{\mathbf{k}, \mathbf{k}^{\prime}} \Phi_{1}\left(\mathbf{k}, \mathbf{k}^{\prime}\right) a_{1 \mathbf{k}}^{\dagger} b_{1 \mathbf{k}^{\prime}}^{\dagger}|G\rangle \\
& =\iint d \mathbf{r} d \mathbf{r}^{\prime} \Psi_{1}\left(\mathbf{r}, \mathbf{r}^{\prime}\right) a_{1 \mathbf{r}^{\dagger}}^{\dagger} b_{1 \mathbf{r}^{\prime}}^{\dagger}|G\rangle
\end{aligned}
$$

with energy $E_{0}+E_{1}$; third, the single-exciton state made up of an electron in $c 2$ band and a hole in $h 2$ band,

$$
\begin{aligned}
|S 2\rangle & =\sum_{\mathbf{k}, \mathbf{k}^{\prime}} \Phi_{2}\left(\mathbf{k}, \mathbf{k}^{\prime}\right) a_{2 \mathbf{k}}^{\dagger} b_{2 \mathbf{k}^{\prime}}^{\dagger}|G\rangle \\
& =\iint d \mathbf{r} d \mathbf{r}^{\prime} \Psi_{2}\left(\mathbf{r}, \mathbf{r}^{\prime}\right) a_{2 \mathbf{r}^{\dagger}}^{\dagger} b_{2 \mathbf{r}^{\prime}}^{\dagger}|G\rangle
\end{aligned}
$$

with energy $E_{0}+E_{2}$; fourth, the biexciton state made up of an electron in $c 1$ band, an electron in $c 2$ band, a hole in $h 1$ band, and a hole in $h 2$ band,

$$
\begin{aligned}
& |B\rangle=\sum_{\mathbf{k}_{1}, \mathbf{k}_{1}^{\prime}, \mathbf{k}_{2}, \mathbf{k}_{2}^{\prime}} \Phi_{B}\left(\mathbf{k}_{1}, \mathbf{k}_{1}^{\prime}, \mathbf{k}_{2}, \mathbf{k}_{2}^{\prime}\right) a_{1 \mathbf{k}_{1}}^{\dagger} b_{1 \mathbf{k}_{1}^{\prime}}^{\dagger} a_{2 \mathbf{k}_{2}}^{\dagger} b_{2 \mathbf{k}_{2}^{\prime}}^{\dagger}|G\rangle \\
& =\iiint \int d \mathbf{r}_{1} d \mathbf{r}_{1}^{\prime} d \mathbf{r}_{2} d \mathbf{r}_{2}^{\prime} \Psi_{B}\left(\mathbf{r}_{1}, \mathbf{r}_{1}^{\prime}, \mathbf{r}_{2}, \mathbf{r}_{2}^{\prime}\right) \\
& \times a_{1 \mathbf{r}_{1}}^{\dagger} b_{1 \mathbf{r}_{1}^{\prime}}^{\dagger} a_{2 \mathbf{r}_{2}}^{\dagger} b_{2 \mathbf{r}_{2}^{\prime}}^{\dagger}|G\rangle
\end{aligned}
$$

with energy $E_{0}+E_{B}$. The wavefunctions of these exciton and biexciton states are the lowest bound state wavefunctions of the corresponding stationary Schrödinger equations.

Now consider the coupling with light. For a light $\mathbf{A}(\mathbf{r})=\eta_{\mathbf{q}}\left(A_{\mathbf{q}} e^{i \mathbf{q} \cdot \mathbf{r}-i \omega_{\mathbf{q}} t}+A_{\mathbf{q}}^{*} e^{-i \mathbf{q} \cdot \mathbf{r}+i \omega_{\mathbf{q}} t}\right)$, where $\eta_{\mathbf{q}}$ is the unit polarization vector, $\mathbf{q} \approx 0$, the electron-light coupling Hamiltonian is

$$
\begin{aligned}
\mathcal{H}_{e q} & =\int \psi^{\dagger}(\mathbf{r}) d \mathbf{r}\left[-\frac{e}{m} \mathbf{p} \cdot \mathbf{A}(\mathbf{r})\right] \psi(\mathbf{r}) d \mathbf{r} \\
& =-\frac{e}{m} \sum_{\mu \nu \mathbf{k}}\left(M_{\mu \nu \mathbf{k q}} a_{\mu \mathbf{k}}^{\dagger} a_{\nu \mathbf{k}} A_{\mathbf{q}} e^{-i \omega_{\mathbf{q}} t}+H . c .\right)
\end{aligned}
$$

where $M_{\mu \nu \mathbf{k q}}=\int_{\text {cell }} d \mathbf{r} u_{\mu \mathbf{k}}^{*}(\mathbf{r}) \mathbf{p} \cdot \eta_{\mathbf{q}} u_{\nu \mathbf{k}}(\mathbf{r})$. This remains unchanged in presence of a magnetic field, which is about constant in the crystal unit cell, since $\int_{\text {cell }} d \mathbf{r} u_{\mu \mathbf{k}}^{*}(\mathbf{r}) u_{\nu \mathbf{k}}=$ 0 .

In the present situation, consider the coupling with two circularly polarized monochromatic lights [4]. One is with $\eta_{\mathbf{q} 1}=\left(-\mathbf{e}_{x}-i \mathbf{e}_{y}\right) / \sqrt{2}, M_{\mu \nu \mathbf{k q} 1} \neq 0$ only for $\mu=c 1$, $\nu=h 1$. Its interaction with electrons is

$$
\mathcal{H}_{e q 1}=-\frac{e}{m} \sum_{\mathbf{k}}\left(M_{c 1 h 1 \mathbf{k q} 1} a_{1 \mathbf{k}}^{\dagger} b_{1-\mathbf{k}}^{\dagger} A_{\mathbf{q} 1} e^{-i \omega_{\mathbf{q} 1} t}+H . c .\right) .
$$

The other light is with $\eta_{\mathbf{q} 2}=\left(\mathbf{e}_{x}-i \mathbf{e}_{y}\right) / \sqrt{2} M_{\mu \nu \mathbf{k q} 2} \neq 0$ only for $\mu=c 2, \nu=h 2$. Its interaction with electrons is

$$
\mathcal{H}_{e q 2}=-\frac{e}{m} \sum_{\mathbf{k}}\left(M_{c 2 h 2 \mathbf{k q} 2} a_{2 \mathbf{k}}^{\dagger} b_{2-\mathbf{k}}^{\dagger} A_{\mathbf{q} 2} e^{-i \omega_{\mathbf{q} 2} t}+H . c .\right) .
$$

With the interaction with these two light modes, the total Hamiltonian is

$$
\mathcal{H}=\mathcal{H}_{e}+\mathcal{H}_{e q 1}+\mathcal{H}_{e q 2}
$$

Under $\mathcal{H}$, the electronic state $|\Psi(t)\rangle$ is determined by

$$
i \hbar \frac{\partial|\Psi(t)\rangle}{\partial t}=\mathcal{H}|\Psi(t)\rangle .
$$

In terms of the four relevant eigenstates of $\mathcal{H}_{e},|\Psi(t)\rangle$ can be expanded as

$$
\begin{aligned}
|\Psi(t)\rangle= & f_{G}|G\rangle+f_{S 1} e^{-i E_{1} t / \hbar}|S 1\rangle+f_{S 2} e^{-i E_{2} t / \hbar}|S 2\rangle \\
& +f_{B} e^{-i E_{B} t / \hbar}|B\rangle,
\end{aligned}
$$

where the coefficients $f_{k}$ are determined by

$i \hbar \frac{\partial f_{k}(t)}{\partial t}=\sum_{n} f_{n}(t) e^{-i\left(E_{n}-E_{k}\right) t / \hbar}\left(\left\langle k\left|\mathcal{H}_{e q 1}\right| n\right\rangle+\left\langle k\left|\mathcal{H}_{e q 2}\right| n\right\rangle\right)$,

as obtained from (7). The initial condition is $f_{G}(0)=1$. 
Note that the only non-vanishing matrix elements of $H_{e q 11}$ and $H_{e q 2}$ are

$$
\begin{aligned}
\left\langle S 1\left|\mathcal{H}_{e q 1}\right| G\right\rangle=- & \frac{e}{m} \sum_{\mathbf{k}} \Phi_{1}^{*}(\mathbf{k},-\mathbf{k}) M_{c 1 h 1 \mathbf{k q} 1} A_{\mathbf{q} 1} e^{-i \omega_{\mathbf{q} 1} t}, \\
\left\langle S 2\left|\mathcal{H}_{e q 2}\right| G\right\rangle=- & \frac{e}{m} \sum_{\mathbf{k}} \Phi_{2}^{*}(\mathbf{k},-\mathbf{k}) M_{c 2 h 2 \mathbf{k q} 2} A_{\mathbf{q} 2} e^{-i \omega_{\mathbf{q} 2} t} \\
\left\langle B\left|\mathcal{H}_{e q 2}\right| S 1\right\rangle= & -\frac{e}{m} \sum_{\mathbf{k}, \mathbf{k}_{1}, \mathbf{k}_{1}^{\prime}} \Phi_{B}^{*}\left(\mathbf{k}_{1}, \mathbf{k}_{1}^{\prime}, \mathbf{k},-\mathbf{k}\right) \\
& \times \Phi_{1}\left(\mathbf{k}_{1}, \mathbf{k}_{1}^{\prime}\right) M_{c 2 h 2 \mathbf{k q} 2} A_{\mathbf{q} 2} e^{-i \omega_{\mathbf{q} 2} t} \\
\left\langle B\left|\mathcal{H}_{e q 1}\right| S 2\right\rangle= & -\frac{e}{m} \sum_{\mathbf{k}, \mathbf{k}_{2}, \mathbf{k}_{2}^{\prime}} \Phi_{B}^{*}\left(\mathbf{k},-\mathbf{k}, \mathbf{k}_{2}, \mathbf{k}_{2}^{\prime}\right) \\
& \times \Phi_{2}\left(\mathbf{k}_{2}, \mathbf{k}_{2}^{\prime}\right) M_{c 1 h 1 \mathbf{k q} 1} A_{\mathbf{q} 1} e^{-i \omega_{\mathbf{q} 1} t}
\end{aligned}
$$

and their transposes.

In the perturbative expansion, $f_{k}=\sum_{j} f_{k}^{(j)}$, where $j$ represents the order of perturbation. Thus, $i \hbar \partial f_{k}^{(j)}(t) / \partial t=\sum_{n} \exp \left[-i\left(E_{n}-E_{k}\right) t / \hbar\right]\left(\left\langle k\left|\mathcal{H}_{e q 1}\right| n\right\rangle+\right.$ $\left.\left\langle k\left|\mathcal{H}_{e q 2}\right| n\right\rangle\right) f_{n}^{(j-1)}$. Therefore, in each term of $f^{(j)}$, there should be a product of $j$ matrix elements of $\mathcal{H}_{e q 1}$ or $\mathcal{H}_{e q 2}$, in terms of $j-1$ intermediate states connecting initial state $|G\rangle$ and state $|k\rangle$., i.e. $\left\langle k\left|H_{1}\right| n_{1}\right\rangle\left\langle n_{1}\left|H_{2}\right| n_{2}\right\rangle \cdots\left\langle n_{j-1}\left|H_{k}\right| G\right\rangle$, where each $H_{i}(i=$ $1, \cdots k)$ is either $\mathcal{H}_{e q 1}$ or $\mathcal{H}_{e q 2}$.

From this, it can be seen that $f_{B}(t)$ approaches zero quickly with time. First, due to angular momentum selection rule, $f_{B}(t)$ exactly vanishes in odd orders, where there must be $\left\langle B\left|\mathcal{H}_{e q 1}+\mathcal{H}_{e q 2}\right| G\right\rangle$, which is zero. Second, it can be seen that given $\hbar \omega_{\mathbf{q} i}=E_{i}$ while $E_{B} \neq E_{1}+E_{2}$ due to Coulomb interaction, each even order, involving integrals over time, approaches zero quickly with time. This is, of course, the offresonance effect. In contrast, $f_{S i}(t)$ is nonvanishing and becomes appreciable for sufficiently long time, because of resonance $\hbar \omega_{\mathbf{q} i}=E_{i}$. The first order result is $f_{S 1}(t) \approx \frac{e}{\hbar m} \sum_{\mathbf{k}} \Phi_{1}^{*}(\mathbf{k},-\mathbf{k}) M_{c 1 h 1 \mathbf{k q} 1} A_{\mathbf{q} 1} \frac{e^{i\left(E_{1} / \hbar-\omega_{\mathbf{q} 1}\right) t}-1}{\omega_{G, S 1}-\omega_{\mathbf{q} 1}}$ (and the similar expression for $f_{S 2}$ ), which can easily lead to the well-known Eliot formula of the transition rate, which is usually derived in a different way.

The point we particularly want to emphasize is that although Coulomb interaction prevents the appearance of $|B\rangle$, it is irrelevant to the situation that interaction of particles is needed to generate entanglement between distinguishable particles. The entanglement in the present case is that of occupation numbers, whose generation depends on the coupling between single particle basis states, which is offered here by the electron-light interaction. In fact, occupation-number entanglement still exists even when the state is a superposition of the ground state and one single-excitonic eigenstate, or even simply in a single-excitonic eigenstate. If Coulomb interaction is negligible, the states generated by the two lights are two independent states, each being a superposition state of the ground state and an single-excitonic eigenstate, in which there exists occupation-number entanglement.
In general, interactions of particles is not necessary unless the single particle basis states are the eigenstates of the single particle Hamiltonian. In the case of optical control, the single particle Hamiltonian which defines the single particle basis does not include the electron-light interaction, which thus couples different single particle basis states.

\section{ENTANGLEMENT CHARACTERIZATION OF THE SUPERPOSITION OF DIFFERENT EXCITONIC STATES}

In the preceding section, we have shown that the state generated must be of the form

$$
|\Psi\rangle=g_{G}|G\rangle+g_{S 1}|S 1\rangle+g_{S 2}|S 2\rangle,
$$

which was obtained in the experiment, as indicated by the interference line shape in the coherent nonlinear response, using the two light modes as pump and probe fields respectively [4].

Now we analyze the entanglement in the state $|\Psi\rangle$ in Eq. (91). In details of the occupation-numbers of the single electron or hole basis states at the four relevant bands,

$$
\begin{aligned}
& |\Psi\rangle=g_{G} \prod_{\mathbf{k}_{1} \mathbf{k}_{1}^{\prime}}|0\rangle_{c 1 \mathbf{k}_{1}}|0\rangle_{h 1 \mathbf{k}_{1}^{\prime}} \prod_{\mathbf{k}_{2} \mathbf{k}_{2}^{\prime}}|0\rangle_{c 2 \mathbf{k}_{2}}|0\rangle_{h 2 \mathbf{k}_{2}^{\prime}} \\
& +g_{S 1} \sum_{\mathbf{k}_{1} \mathbf{k}_{1}^{\prime}} \Phi_{1}\left(\mathbf{k}_{1}, \mathbf{k}_{1}^{\prime}\right)|1\rangle_{c 1 \mathbf{k}_{1}}|1\rangle_{h 1 \mathbf{k}_{1}^{\prime}} \\
& \times \prod_{\overline{\mathbf{k}}_{1} \overline{\mathbf{k}}_{1}^{\prime}}|0\rangle_{c 1 \overline{\mathbf{k}}_{1}}|0\rangle_{h 1 \overline{\mathbf{k}}_{1}^{\prime}} \prod_{\mathbf{k}_{2} \mathbf{k}_{2}^{\prime}}|0\rangle_{c 2 \mathbf{k}_{2}}|0\rangle_{h 2 \mathbf{k}_{2}^{\prime}} \\
& +g_{S 2} \prod_{\mathbf{k}_{1} \mathbf{k}_{1}^{\prime}}|0\rangle_{c 1 \mathbf{k}_{1}}|0\rangle_{h 1 \mathbf{k}_{1}^{\prime}} \\
& \times \sum_{\mathbf{k}_{2} \mathbf{k}_{2}^{\prime}} \Phi_{2}\left(\mathbf{k}_{2}, \mathbf{k}_{2}^{\prime}\right)|1\rangle_{c 2 \mathbf{k}_{2}}|1\rangle_{h 2 \mathbf{k}_{2}^{\prime}} \prod_{\overline{\mathbf{k}}_{2} \overline{\mathbf{k}}_{2}^{\prime}}|0\rangle_{c 2 \overline{\mathbf{k}}_{2}}|0\rangle_{h 2 \overline{\mathbf{k}}_{2}^{\prime}},
\end{aligned}
$$

where $\overline{\mathbf{k}}_{i} \neq \mathbf{k}_{i}, \overline{\mathbf{k}}_{i}^{\prime} \neq \mathbf{k}_{i}^{\prime}$.

As explained in Sec. II, in the present case, the subsystems are single particle basis states, and the reduced density matrices and the entanglement are those of occupation-numbers. For example, the reduced density matrix of the occupation-number of $\left|c i \mathbf{k}_{i}\right\rangle$ is

$$
\left\langle n\left|\rho_{c i \mathbf{k}_{i}}\right| n^{\prime}\right\rangle=\sum_{n_{l} \cdots n_{\infty}}\left\langle n, n_{l} \cdots n_{\infty} \mid \Psi\right\rangle\left\langle\Psi \mid n^{\prime}, n_{l} \cdots n_{\infty}\right\rangle
$$

where $l, \cdots, \infty$ represent all the single particle basis states other than $\left|c i \mathbf{k}_{i}\right\rangle$.

The entanglement between $\left|c i \mathbf{k}_{i}\right\rangle$ and the rest of the system is thus, as the von Neumann entropy of (10)

$$
S_{c i \mathbf{k}_{i}}=-\alpha_{i \mathbf{k}_{i}} \ln \alpha_{i \mathbf{k}_{i}}-\left(1-\alpha_{i \mathbf{k}_{i}}\right) \ln \left(1-\alpha_{i \mathbf{k}_{i}}\right) .
$$

This is obtained by considering $\left\langle 1\left|\rho_{c i \mathbf{k}_{i}}\right| 1\right\rangle=\alpha_{i \mathbf{k}_{i}}=$ $\left|g_{S i}\right|^{2} \sum_{\mathbf{k}_{i}^{\prime}}\left|\Phi_{i}\left(\mathbf{k}_{i}, \mathbf{k}_{i}^{\prime}\right)\right|^{2},\left\langle 0\left|\rho_{c i \mathbf{k}_{i}}\right| 0\right\rangle=1-\alpha_{i \mathbf{k}_{i}}$, and that $\rho_{c i \mathbf{k}_{i}}$ is diagonal in the basis $(|0\rangle,|1\rangle)$, basically for the reason that whenever $\left|c i \mathbf{k}_{i}\right\rangle$ is occupied, there is always an occupied hole band state. Similarly, the entanglement between $\left|h i \mathbf{k}_{i}^{\prime}\right\rangle$ and the rest of the system is

$$
S_{h i \mathbf{k}_{i}^{\prime}}=-\alpha_{i \mathbf{k}_{i}^{\prime}} \ln \alpha_{i \mathbf{k}_{i}^{\prime}}-\left(1-\alpha_{i \mathbf{k}_{i}^{\prime}}\right) \ln \left(1-\alpha_{i \mathbf{k}_{i}^{\prime}}\right)
$$


where $\alpha_{i \mathbf{k}_{i}^{\prime}}=\left|g_{S i}\right|^{2} \sum_{\mathbf{k}_{i}}\left|\Phi_{i}\left(\mathbf{k}_{i}, \mathbf{k}_{i}^{\prime}\right)\right|^{2}$. The entanglement between $\left|c i \mathbf{k}_{i}\right\rangle\left|h i \mathbf{k}_{i}^{\prime}\right\rangle$ and the rest of the system can be calculated to be

$$
\begin{aligned}
S_{c i \mathbf{k}_{i}, h i \mathbf{k}_{i}^{\prime}}= & -\left|g_{S i} \Phi_{i}\left(\mathbf{k}_{i}, \mathbf{k}_{i}^{\prime}\right)\right|^{2} \ln \left|g_{S i} \Phi_{i}\left(\mathbf{k}_{i}, \mathbf{k}_{i}^{\prime}\right)\right|^{2} \\
& -\gamma_{i \mathbf{k}_{i}} \ln \gamma_{i \mathbf{k}_{i}}-\gamma_{i \mathbf{k}_{i}^{\prime}} \ln \gamma_{i \mathbf{k}_{i}^{\prime}} \\
& -\left(1-\gamma_{i \mathbf{k}_{i}}-\gamma_{i \mathbf{k}_{i}^{\prime}}\right) \ln \left(1-\gamma_{i \mathbf{k}_{i}}-\gamma_{i \mathbf{k}_{i}^{\prime}}\right)
\end{aligned}
$$

where $\gamma_{i \mathbf{k}_{i}}=\left|g_{S i}\right|^{2} \sum_{\mathbf{q}_{i}^{\prime} \neq \mathbf{k}_{i}^{\prime}}\left|\Phi_{i}\left(\mathbf{k}_{i}, \mathbf{k}_{i}^{\prime}\right)\right|^{2}, \quad \gamma_{i \mathbf{k}_{i}^{\prime}}=$ $\left|g_{S i}\right|^{2} \sum_{\mathbf{q}_{i} \neq \mathbf{k}_{i}}\left|\Phi_{i}\left(\mathbf{k}_{i}, \mathbf{k}_{i}^{\prime}\right)\right|^{2}$.

Note that these three results are valid no matter whether the $g_{G}$ and $g_{S j}(j \neq i)$ are 0 or not, which only affects the value of $1-\alpha_{i \mathbf{k}_{i}}$ and $1-\alpha_{i \mathbf{k}_{i}^{\prime}}$. When $g_{S j}=0$, no matter whether $g_{G}=0$, the single particle basis states with index $j$ become separated out. Replacing $\Phi_{i}\left(\mathbf{k}_{i}, \mathbf{k}_{i}^{\prime}\right)$ by $\Psi_{i}(\mathbf{r}, \mathbf{r})$, one obtains entanglements concerning $\mid$ cir $\rangle$ and $\left|h i \mathbf{r}^{\prime}\right\rangle$, i.e. when the modes are defined by positions rather than wave vectors.

When and only when $g_{G}=0$ while both $g_{S 1}$ and $g_{S 2}$ are nonzero, the nature of entanglement can be accounted in terms of two existing distinguishable particles: one electron and one hole. In the present case, the basis states of the electron and the hole are spinors. There are two degrees of freedoms, the band index (i.e. angular momentum and effective mass) and the orbit (position or wavevector). The effective state of the two distinguishable particles is

$$
\begin{aligned}
& |\Psi\rangle=\sum_{\mathbf{k}, \mathbf{k}^{\prime}}\left[g_{S 1} \Phi_{1}\left(\mathbf{k}, \mathbf{k}^{\prime}\right)|c 1\rangle|h 1\rangle\right. \\
& \left.+g_{S 2} \Phi_{2}\left(\mathbf{k}, \mathbf{k}^{\prime}\right)|c 2\rangle|h 2\rangle\right] \otimes|\mathbf{k}\rangle\left|\mathbf{k}^{\prime}\right\rangle \\
& =\iint\left[g_{S 1} \Psi_{1}\left(\mathbf{r}, \mathbf{r}^{\prime}\right)|c 1\rangle|h 1\rangle\right. \\
& \left.+g_{S 2} \Psi_{2}\left(\mathbf{r}, \mathbf{r}^{\prime}\right)|c 2\rangle|h 2\rangle\right] \otimes|\mathbf{r}\rangle\left|\mathbf{r}^{\prime}\right\rangle d^{3} r d^{3} r^{\prime}
\end{aligned}
$$

where $|c i\rangle, \mathbf{k}$ and $\mathbf{r}$ are for the electron, $|h i\rangle, \mathbf{k}^{\prime}$ and $\mathbf{r}^{\prime}$ are for the hole. The reduced density matrix of the hole,

$$
\begin{aligned}
\rho_{h} & \equiv \sum_{\mathbf{k}} \sum_{i}\langle c i|\langle\mathbf{k} \mid \Psi\rangle\langle\Psi \mid \mathbf{k}\rangle| c i\rangle \\
& \equiv \sum_{i} \int d^{3} r\langle c i|\langle\mathbf{r} \mid \Psi\rangle\langle\Psi \mid \mathbf{r}\rangle| c i\rangle
\end{aligned}
$$

is

$$
\begin{gathered}
\sum_{\mathbf{k}, \mathbf{k}^{\prime}, \mathbf{k}^{\prime \prime}, i}\left|g_{S i}\right|^{2} \Phi_{i}\left(\mathbf{k}, \mathbf{k}^{\prime}\right) \Phi_{i}^{*}\left(\mathbf{k}, \mathbf{k}^{\prime \prime}\right)|h i\rangle\left\langle h i|\otimes| \mathbf{k}^{\prime}\right\rangle\left\langle\mathbf{k}^{\prime \prime}\right| \\
=\sum_{i} \int d^{3} r \int d^{3} r^{\prime} \int d^{3} r^{\prime \prime}\left|g_{S i}\right|^{2} \Psi_{i}\left(\mathbf{r}, \mathbf{r}^{\prime}\right) \Psi_{i}^{*}\left(\mathbf{r}, \mathbf{r}^{\prime \prime}\right) \\
\times|h i\rangle\left\langle h i|\otimes| \mathbf{r}^{\prime}\right\rangle\left\langle\mathbf{r}^{\prime \prime}\right|
\end{gathered}
$$

. The entanglement between the electron and the hole is quantified to be $S_{h}=-\operatorname{tr} \rho_{h} \ln \rho_{h}$.

The orbital state, obtained by tracing out the band indices, is

$$
\begin{aligned}
\rho^{\text {orbits }}= & \sum_{\mathbf{k}, \mathbf{k}^{\prime}}\left[\left|g_{S 1}\right|^{2}\left|\Phi_{1}\left(\mathbf{k}, \mathbf{k}^{\prime}\right)\right|^{2}+\left|g_{S 2}\right|^{2}\left|\Phi_{2}\left(\mathbf{k}, \mathbf{k}^{\prime}\right)\right|^{2}\right] \\
= & \iint_{\mathbf{k}\rangle\left\langle\mathbf{k}|\otimes| \mathbf{k}^{\prime}\right\rangle\left\langle\mathbf{k}^{\prime}\right|}\left[\left|g_{S 1}\right|^{2}\left|\Psi_{1}\left(\mathbf{r}, \mathbf{r}^{\prime}\right)\right|^{2}+\left|g_{S 2}\right|^{2}\left|\Psi_{2}\left(\mathbf{r}, \mathbf{r}^{\prime}\right)\right|^{2}\right] \\
& \times|\mathbf{r}\rangle\left\langle\mathbf{r}|\otimes| \mathbf{r}^{\prime}\right\rangle\left\langle\mathbf{r}^{\prime}\right| d^{3} r d^{3} r^{\prime},
\end{aligned}
$$

which is presumably a bipartite mixed state of continuous variables, with each part living in an infinite dimensional Hilbert space, for which there is not yet an analytical entanglement measure.
The most interesting and experimentally detectable entanglement, which is indeed the one detected in the [4], is that between the band indices, after the Bloch wavevector or position wavefunction is traced out. The density matrix of the band-index state is thus

$$
\begin{aligned}
\rho^{\text {bands }}= & \left|g_{S 1}\right|^{2}|h 1\rangle\langle h 1|\otimes| c 1\rangle\langle c 1| \\
& +g_{S 1} g_{S 2}^{*} x|h 1\rangle\langle h 2|\otimes| c 1\rangle\langle c 2| \\
& +g_{S 2} g_{S 1}^{*} x^{*}|h 2\rangle\langle h 1|\otimes| c 2\rangle\langle c 1| \\
& +\left|g_{S 2}\right|^{2}|h 2\rangle\langle h 2|\otimes| c 2\rangle\langle c 2|,
\end{aligned}
$$

where $x \equiv \sum_{\mathbf{k}, \mathbf{k}^{\prime}} \Phi_{1}\left(\mathbf{k}, \mathbf{k}^{\prime}\right) \Phi_{2}^{*}\left(\mathbf{k}, \mathbf{k}^{\prime}\right) \quad \equiv$ $\int \Psi_{1}\left(\mathbf{r}, \mathbf{r}^{\prime}\right) \Psi_{2}^{*}\left(\mathbf{r}, \mathbf{r}^{\prime}\right) d^{3} r d^{3} r^{\prime}$ is the overlap between the wavefunctions of the two excitonic eigenstates. Presumably, $\rho^{\text {bands }}$, like $\rho^{\text {orbits }}$, is also a mixed state.

However, interestingly

$$
\Psi_{1}\left(\mathbf{r}, \mathbf{r}^{\prime}\right)=\Psi_{2}\left(\mathbf{r}, \mathbf{r}^{\prime}\right) \equiv \Psi\left(\mathbf{r}, \mathbf{r}^{\prime}\right),
$$

and

$$
\Phi_{1}\left(\mathbf{k}, \mathbf{k}^{\prime}\right)=\Phi_{2}\left(\mathbf{k}, \mathbf{k}^{\prime}\right) \equiv \Phi_{\left(\mathbf{k}, \mathbf{k}^{\prime}\right),}
$$

because both $\Psi_{1}$ and $\Psi_{2}$, or both $\Phi_{1}$ and $\Phi_{2}$, are the lowest bound state wavefunctions, which is independent of the effective masses, which only affect the energy. Hence $|x|=1$.

Therefore, both $\rho^{\text {orbits }}$ and $\rho^{\text {bands }}$ become pure states. In other words, in the state $g_{S 1}|S 1\rangle+g_{S 2}|S 2\rangle$, band index and orbital degrees of freedom become separable. Consequently, the total entanglement between the electron and the hole is the sum of the entanglement in the orbital and that in band-index states.

The orbital state is $\rho^{\text {bands }}=|\phi\rangle\langle\phi|$, with

$$
\begin{aligned}
|\phi\rangle & =\sum_{\mathbf{k}, \mathbf{k}^{\prime}} \Phi\left(\mathbf{k}, \mathbf{k}^{\prime}\right)|\mathbf{k}\rangle\left|\mathbf{k}^{\prime}\right\rangle \\
& =\iint \Psi\left(\mathbf{r}, \mathbf{r}^{\prime}\right)|\mathbf{r}\rangle\left|\mathbf{r}^{\prime}\right\rangle d \mathbf{r} d \mathbf{r}^{\prime},
\end{aligned}
$$

in which the entanglement is quantified as the von Neumann entropy of the reduced density matrix of either the electron or the hole obtained from the orbital wavefunction.

The band index state is $\rho^{\text {bands }}=|\psi\rangle\langle\psi|$, with

$$
|\psi\rangle=g_{S 1}|c 1\rangle|h 1\rangle+g_{S 2}|c 2\rangle|h 2\rangle .
$$

Hence the band-index entanglement between the electron and the hole is

$$
S^{\text {bands }}=-\left|g_{S 1}\right|^{2} \ln \left|g_{S 1}\right|^{2}-\left|g_{S 2}\right|^{2} \ln \left|g_{S 2}\right|^{2} .
$$

A speciality here is that the state is a superposition of two eigenstates with different angular momenta, which can be probed by using magnetic field, as well as different effective masses, which can be probed by using cyclotron resonance.

The factorization, or disentanglement, of band index and orbital states as realized in this state, is very interesting for quantum computing in semiconductors. If the spin is used as qubit, spin-orbit coupling causes decoherence and error. But if the total angular momentum is used as qubit, spin-orbit coupling may not cause decoherence, as exemplified by the study here. 


\section{SPATIAL SEPARATION}

We can spatially separate the electron and hole by engineering the orbital envelope wavefunctions of the excitonic eigenstate. The spatial separation is of significant interest in quantum information and quantum foundations. Note that the band-index, or angular-momentum, is not coupled to the external barrier or electric field which are used in engineer the orbital wavefunction.

If the orbital degree of freedom is "entangled" with the band index, then engineering orbital wavefunction also influences the band index state. Moreover, it causes problem in whether one can measure band index state $\rho^{\text {bands }}$, which is obtained by tracing out the orbital degree of freedom. This could be a source of decoherence of the band-index state. Other sources of decoherence include the phonons, nuclear spins, etc.

However, as discussed in the preceding section, in the state $g_{S 1}|S 1\rangle+g_{S 2}|S 2\rangle$, band index and orbital degrees of freedom are separated. Hence in spatially separating the electron and hole, the angular momentum, i.e. the band index state is not influenced, simply like the case of spatially separating an Einstein-Podolsky-Rosen-Bohm pair which is spin-entangled. Also, it of course does not matter if the state has a ground-state component, which is simply not affected. Therefore spin-orbit coupling does not cause decoherence in the band-indices or angular momenta in the state $|\Psi\rangle=g_{G}|G\rangle+g_{S 1}|S 1\rangle+g_{S 2}|S 2\rangle$.

In the following, we suggest a few methods of achieving spatial separation, by exploiting various physical properties of semiconductor heterostructures [18]. One method is to let the quantum dot or well, in which the electronhole pair is generated, tunnel-couple with another one or more dots or wells. When the size of the dot or well is no smaller than the radius of the two excitonic eigenstates, the optical generation is not affected. After generation, tunnelling gives rise to probability of finding electron and hole in different dots.

It is intriguing to give some detail of the tunnelling of the entangled state. The total Hamiltonian is $H=\mathcal{H}_{A}+$ $\mathcal{H}_{B}+H_{T}$, where $\mathcal{H}_{A}$ and $\mathcal{H}_{B}$ are electronic Hamiltonians in the two dots. The tunnelling Hamiltonian is

$$
\begin{aligned}
& H_{T}=\sum_{i} \sum_{\mathbf{k}_{\mu} \mathbf{k}_{\nu}}\left(t_{c i \mathbf{k}_{\mu} \mathbf{k}_{\nu}} a_{i \mathbf{k}_{\mu}}^{A}{ }^{\dagger} a_{i \mathbf{k}_{\nu}}^{B}+H . c\right) \\
& +\sum_{i} \sum_{\mathbf{k}_{\mu} \mathbf{k}_{\nu}}\left(t_{h i \mathbf{k}_{\mu} \mathbf{k}_{\nu}}^{\prime} b_{i-\mathbf{k}_{\mu}}^{A}{ }^{\dagger} b_{i-\mathbf{k}_{\nu}}^{B}+H . c\right) \text {. }
\end{aligned}
$$

Because it does not change the band index, tunnelling changes the overall state through the change of the envelope function of each excitonic eigenstate independently, from an excitonic bound state to a superposition including the component in which the electron and hole reside in different dots. Suppose the optically generated state is given by (9). With tunnelling, the state can still be written in the form of (9), with only the orbital wavefunctions of $|S 1\rangle$ and $|S 2\rangle$ transformed. The band-index state remains unaffected. If during the tunnelling, the magnetic field which removes the band degeneracy is present or absent in both dots or wells, then the two conduction bands see a same barrier, and the two hole bands also see a same barrier. Consequently, given $\Psi_{1}\left(\mathbf{r}, \mathbf{r}^{\prime}\right)$ and $\Psi_{2}\left(\mathbf{r}, \mathbf{r}^{\prime}\right)$ are equal initially, they remain equal under tunnelling, though each becomes a delocalized superposition. When $g_{G}=0$, this gives rise to spatial separation of the electron and the hole in the band-index pure state (111). Remember there is only one pair of electron and hole, which is in a superposition state before measurement or decoherence.

Furthermore, an electric field can localize electron and hole in different dots or wells, due to WannierStark effect. Consequently, $\Psi_{1}\left(\mathbf{r}, \mathbf{r}^{\prime}\right)=\Psi_{2}\left(\mathbf{r}, \mathbf{r}^{\prime}\right)$ becomes $\phi_{A}(\mathbf{r}) \phi_{B}\left(\mathbf{r}^{\prime}\right)$. The electric field may either be exerted after optical generation or be present even during the optical generation. The latter option, however, shifts the resonant energies and, when the field is strong enough, causes ionization, which then brings in the bi-excitonic component. Note that electric field does not couple to the band-index (angular momentum) degree of freedom, and that the band-index state is separated from the orbital state, therefore the electric field does not cause the decoherence of the band-index state.

It is interesting to study entangled electron-hole state in a superlattice, i.e. many coupled quantum wells. Without electric field, each excitonic state is delocalized over a large region. By using an electric field, localization of electron and hole with large spatial separation can be achieved, allowing various studies of entanglement properties and quantum informational process.

Another method is to make the two excitonic eigenstates indirect in real space, i.e. electron and hole are confined in different sides of the heterojunction. As is well known, this can be achieved by the so-called type-II heterojunctions, in which the lower conduction band and the higher hole band are on the two different sides. Such an interface is formed by III-V compounds with both different group III elements and different group V elements.

\section{SUMMARY}

To summarize, in the framework of quantum field theory, we studied characterizations and optical generation of entanglement in an electron-hole system, with the consideration of spin-orbit coupling, and have given a theoretical account of an interesting experimental result [4]. For a many-electron system, different single particle states are distinguishable subsystems. The entanglement is between occupation-numbers of different single particle states, and is generated when the field theoretic Hamiltonian couples different single particle basis states.

For a semiconductor, coupling with two resonant light modes of different circular polarizations leads to a superposition of ground state and the two different single excitonic eigenstates, each of which is made up of an electron and a hole in the corresponding conduction and heavy-hole bands. In this state, there exists complicated occupation-number entanglement, which we have 
analyzed in detail.

The Coulomb interaction is not essential in generating the occupation-number entanglement. Occupationnumber entanglement also exists in each excitonic eigenstate, as well as its superposition with the ground state.

When the state is a superposition of only the two single-excitonic states, the entanglement can be accounted as between two distinguishable particles, each with two degrees of freedom, band index and the orbital degree of freedom. We find that in this state, tracing out the orbital degree of freedom leads to a pure entangled state in band-index, and vice versa. Hence in this case, the band-index and orbital degrees freedom are separated or non-entangled, despite the spin-orbit coupling in the Hamiltonian. This finding is interesting for quantum computing in semiconductors. It suggests that the problem of spin decoherence due to spin-orbit coupling may be avoided by using the total angular momenta to encode quantum information.

We also briefly propose several methods to spatially separate the electron and the hole, which makes the band-index entanglement nonlocal and thus allows further manipulations. Band-index entanglement means entanglement in both angular momenta and effective masses. This speciality is a consequence of spin-orbit coupling, hence is a manifestation of relativistic effect on quantum entanglement, which is also studied in a different context [19].

Finally, we mention that our method of characterizing the entanglement and its generation in electron-hole systems can equally be applied to the processes proposed in Ref. 6].

\section{Acknowledgments}

I am grateful to Peter Littlewood and Tony Leggett for useful discussions and suggestions.
[1] E. Einstein, B. Podolsky and N. Rosen, Phys. Rev. 47, 777 (1935); E. Schrödinger, Proc. Camb. Phi. Soc. 31, 555 (1935); D. Bohm, Quantum Theory (Prentice-Hall, Englewood Cliffs, 1951); J. S. Bell, Physics 1, 195 (1964).

[2] For reviews, see, e.g. D. Bouwmeester, A. Ekert and A. Zeilinger (ed.), The Physics of Quantum Information (Springer,2000).

[3] J. C. Egues et al., in "Quantum Noise in Mesoscopic Physics", NATO Science Series, vol. 97 (Kluwer, Netherlands, 2003), and references therein; T. Martin, A. Crepieux, and N. Chtchelkatchev, in Proceedings of the NATO ARW workshop on Quantum Noise, ed. Y. Nazarov and Y. Blanter (Kluwer, Netherlands, 2002), and references therein. G. B. Lesovik, T. Martin and G. Blatter, Eur. Phys. J. B 24, 287 (2001). A. T. Costa Jr. and S. Bose, Phys. Rev. Lett. 87, 277901 (2001). W. D. Oliver, F. Yamaguchi, and Y. Yamamoto, Phys. Rev. Lett. 88, 037901 (2002). C. Bena, S. Vishveshwara, L. Balents and M. P. A. Fisher, Phys. Rev. Lett. 89, 037901 (2002). P. Samuelsson, E. V. Sukhorukov and M. Büttiker, Phys. Rev. Lett. 91, 157002 (2003).

[4] G. Chen et al., Science 289, 1906 (2000).

[5] U. Hohenester, Phys. Rev. B 66, 245323 (2002).

[6] C. W. J. Beenakker et al., Phys.Rev.Lett. 91, 147901 (2003).

[7] M. Bayer et al., Science 291, 451 (2001). Y. N. Chen, D. S. Chu and T. Brandes, Phys. Rev. Lett. 90, 166802 (2003).

[8] P. Chen, C. Piermarocchi and L. J. Sham, cond-mat/0009307 P. Chen, C. Piermarocchi and L. J. Sham, Phys. Rev. Lett. 87, 067401 (2001). C. Piermarocchi et al., Phys. Rev. B 65, 075307 (2002). P. Chen et al., cond-mat/0301422
[9] A. Imamoglu et al., Phys. Rev. Lett. 83, 4204 (1999). F. Troiani, U. Hohenester and E. Molinari, Phys. Rev. B 62 R2263 (2000). E. Biolatti et al. Phys. Rev. Lett. 85, $5647(2000)$.

[10] L. Quiroga and N. F. Johnson, Phys. Rev. Lett. 83, 2270 (1999). J. H. Reina, N. F. Johnson, Phys. Rev. A 63, 012303 (2000). F. J. Rodriguez, L. Quiroga, N. F. Johnson, Physica Status Solidi (a) 178 (1), 403-407 (2000). J. H. Reina, Luis Quiroga, N. F. Johnson, Phys. Rev. A 62, 12305 (2000). P. Zhang et al., Phys. Rev. A 67, 012312 (2003).

[11] X. Li et al., Science 301, 809 (2003).

[12] Y. Shi, quant-ph/0204058 J. Phys. A 37, 6807 (2004); quant-ph/0205069 Phys. Lett. A 309, 254 (2003).

[13] Y. Shi, quant-ph/0205069, Phys. Rev. A 67, 024301 (2003).

[14] P. Zanardi, Phys. Rev. A. 65, 042101 (2002).

[15] S. J. van Enk, Phys. Rev. A 67, 022303 (2003).

[16] Use of parametric down-conversion is reviewd by $\mathrm{N}$. Gisin, J. G. Rarity and G. Weihs, in [2]; Y. H. Shih, Rep. Prog. Phys. 66, 1009 (2003). Use of linear optics is discussed in E. Knill, R. Laflamme and G. J. Milburn, Nature 409, 46 (2001).

[17] C. H. Bennett, H. J. Bernstein, S. Popescu and B. Schumacher, Phys. Rev. A 53, 2046 (1996).

[18] For example, P. K. Basu, Theory of Optical Processes in Semiconductors (Clarendon Press, Oxford, 1997).

[19] A. Peres and D. R. Terno, quant-ph/0212023 A. Peres, P. F. Scudo and D. R. Terno, Phys. Rev. Lett. 88, 230402 (2002). P. M. Alsing and G. J. Milburn, quant-ph/0203051 R. M. Gingrich and C. Adami, Phys. Rev. Lett. 89, 270402 (2002). 